\title{
One ICD-10 diagnosis- different conflicts: Using the OPD-CA as tool for treatment planning
}

\author{
Inge Seiffge-Krenke* \\ Department of Psychology, University of Mainz, Germany
}

\begin{abstract}
The diagnostic assessment with DSM-5 or ICD-10 of patients is necessary in psychiatry and psychotherapy, but there are also other important concepts that are necessary the indication and treatment planning. The following article describes the diagnosis based on the OPD-CA, in which, among other things, allows to assess intrapsychic conflicts that impede development and need to be dealt with in therapy. Two cases are presented that have the same diagnosis, F93, but have very different psychodynamic conflicts and also different therapy requirements. The consequences for the subsequent therapy are discussed.
\end{abstract}

\section{Introduction}

The currently available nosological classifications, such as the ICD-10 or DSM-5, are based on symptoms, e.g. clinical disorders were grouped according to symptoms. For diagnostic and clinical work in child and adolescent psychiatry and psychotherapy, it is important to have additional information, such as unconscious conflicts that affect the lives of children and adolescents and inhibit development, the structural requirements and the motivation for therapy. They contain important information for an indication for inpatient or outpatient therapy and guide the further therapeutic approach, e.g. the decision of a conflict vs structure focus in therapy.

Unconscious conflicts are characterized as temporally persistent, contradictory perspectives of feelings and experiences, which the individual tries to integrate by using an active or passive mode. Oriented towards psychoanalytical concepts, the Operationalized Psychodynamic Diagnostics in Childhood and Adolescence (OPDCA-2, 2017) assumes an intrapsychic, long-term conflict as the cause of disorders, in which irreconcilable efforts collide and were "solved" in an active or passive mode. According to OPD-CA, seven conflict issues can be measured: closeness versus distance, submission versus control, taking care of oneself versus being cared for, self-worth conflict, guilt conflict, oedipal conflict, and an identity conflict. The decisive criterion is that the intrapsychic conflict can be observed in various areas of everyday life and inhibits or blocks the further development of the child or adolescent. The integration of these contradicting endeavors does not succeed and colors relationships in different areas of everyday life (e.g. school, family, peers), whereby a distinction is made between an active or passive mode of processing the respective conflict [1].

\section{Two case studies}

The following, we describe two patients with the same diagnosis, F 93.0 Emotional disorder with separation anxiety in childhood, in whom an organic sleep disorder was excluded and whose development was impaired by a different conflict.
F 93.0 Emotional disorder with separation anxiety in childhood based on a conflict taking care of oneself versus being cared for

It is a family in which the mother is so absorbed by her job and no longer has eyes for the daughter. The daughter literally forces her mother to be closer due to symptoms such as sleep disorders and separation anxiety. This does not always lead to the desired success.

\section{The first contact}

9-year-old Maud is introduced by her father. She had just finished a one-week inpatient stay at the children's clinic, because in the evening she heard voices telling her not to fall asleep, otherwise "bad things" would happen. A psychosis could be excluded. The stay in the clinic was the preliminary climax of an increasing problem of falling asleep that has been going on for almost two years. At the beginning there were only a few days on which Maud was unable to fall asleep; since about 3 months she had been afraid of falling asleep every day in the early evening. The fear is so great that Maud has already sat in school with her hair wet, so that the teachers contacted the father and asked what was going on, otherwise Maud is a good student.

The whole family is currently sleeping in the parents' bedroom, which has improved the situation with regard to the problem of falling asleep. The father could not understand all this and was very worried, after all, Maud was otherwise a very self-confident and sporty girl, which the parents would also appreciate very much about her.

${ }^{\star}$ Correspondence to: Inge Seiffge-Krenke, Department of Psychology, University of Mainz, Germany, E-mail: Seiffge.Krenke@uni-main.de

Key words: psychiatry, psychotherapy, psychodynamic diagnosis with OPD-CA, psychodynamic conflicts, F93

Received: November 14, 2020; Accepted: November 23, 2020; Published: November 26, 2020 


\section{Some biographical background}

Maud was breastfed for 4 months, then from one day to the next the mother began to work full time again and was away a lot, often for days, that would have increased more and more over the years. Since Maud was a baby, the non-working father has been looking after her and her younger sister (6 years). In contrast to the sister, who outwardly shows rather stereotypical girlish characteristics and behavior, Maud is seen as very courageous, tough and "adult".

The family moved very often due to the mother's job. In Maud's first year of life, the family moved twice, then they went abroad and, after Maud had attended various kindergartens and finally an international school, returned to Germany; but it seems to be the case that a move abroad is due again soon. At the age of 8 , Maud had problems falling asleep for the first time abroad, and this worsened considerably. Subsequently, the mother was again transferred to Germany, which meant a change of school for Maud, which she found very difficult.

\section{Diagnostic interviews with the patient and her family}

In the interview with Maud, a mature-looking, linguistically fluent, and well-structured girl appears. The clothing shows clear analogies to that of the father, sporty and functional. Her fingernails are badly chewed. She expresses herself in a factual manner about her problems falling asleep and would like the fears to go away. She describes herself as self-confident and sporty, with very good school grades, this weakness just doesn't suit her. Maud seems adjusted and conscientious. While she is painting, she reports that she has found the many moves in recent years "really stupid" and that she very much wishes to stay in one place for a longer period of time. She is also often sad when the mother is absent for several days due to work, which is very common.

After a lot of back and forth about appointments, the mother appears, who is emotionally extremely distant and, when it comes to her daughter's difficulties, seems inappropriately objective and cool. She does not seem to have any understanding for the emotional need of her daughter, emphasizes the good school performance, the athletic fitness of the daughter (she plays soccer) and her many interests and feels visibly annoyed. As soon as she shows up at home, the daughter asked her to lie down to sleep with her. The father, on the other hand, who seems more understanding and tries as best he can to "replace" the mother with the children, now feels overwhelmed (he sleeps with Maud and tries to calm her down when the mother is not there) and doesn't know what to do next. The criticism of his wife is little hidden.

\section{Psychodynamics and passive and active modes of taking care of oneself versus being cared for}

In the conflict Taking care of oneself versus Being cared for principle two modes are possible, an active mode of self-sufficiency and sacrifice for others, and the passive mode of a clinging, parasitic behavior. Maud shows both sides of this conflict. She is pseudo-autonomous during the day, but clearly shows her fear of losing objects at night and ensures the presence of the mother or both parents through the symptom. Here she reacted in the passive mode. The situation of emotional deficiency on the part of the mother, who is emotionally cool and often absent, has been aggravated by the lack of stability in the environment (frequent changes of residence) and the associated changes to kindergarten, school and friends. The symptom is therefore used to secure a piece of "mothering" at least at night. The sad and angry affects experienced by the patient were not recognized by the relatively affect-isolated parents and were therefore not adequately contained; the patient was left alone with her fear and anger. The defense in the form of a counterphobic, ambitious and extremely persistent behavior and the identification with the father helped Maud to counterbalance negative affects stemming from her relationship with her mother. The active mode of taking care the conflict can be seen as well: She takes care of herself in many domains.

\section{F 93.0 Emotional disorder with separation anxiety of childhood based on a submission-control conflict}

Another conflict issue in its development-hindering function is shown in the following patient, who has also developed a sleep disorder and cannot separate from both parents, but especially from the mother

\section{The first contact}

10-year-old Elena is introduced by her mother. Her daughter is emotionally very stressed, no longer sleeps alone, has nightmares and severe fear of separation. For a year she has been very afraid that something could happen to her parents. The parents have therefore reduced their social activities. Elena "always has to be there or I have to inform her where I am and when I will be coming", says the mother. That has increased in the last few months and the parents cannot explain it. She had not slept in her own bed for about 6 months and could only fall asleep if the mother or father lay down with her and stayed with her. Elena is currently sleeping in the double bed. "If she doesn't get her way, Elena screams everything together until she falls asleep from exhaustion". The whole family suffers from the fears that the daughter has developed. "Since Elena sometimes only falls asleep with me, my husband and I have no privacy at all and our relationship suffers from this. We fight a lot. "A few weeks ago, Elena and her father spent a fatherdaughter" weekend in Paris. They shared the "big marriage bed" and Elena was able to fall asleep without difficulty and "enjoyed the time with her father", continues the mother. In addition, fear for her parents makes her want to spend most of the time with them. "Sometimes I have the feeling that Elena wants to own me," continues the mother.

\section{Some biographical information}

Elena was a desired child, was breastfed for 14 months and when she was 15 months, she entered the crawling group, where it was very difficult for her to separate from her mother. However, the transition to kindergarten went smoothly. She is currently in the 4th grade of elementary school. The teachers describe Elena as a smart and "bright" child, always very hardworking and orderly; she has good contact with the teachers and her classmates. In conflicts, however, she never defends herself and does not get angry. "You can't think of staying overnight with friends, Elena is embarrassed that she sleeps in bed with us," says the mother. The parents live in a house with Elena. The patient is an only child. The mother has been working part-time in the office since Elena was 15 months old, the father working full-time as a pharmacist. Due to the parents' work situation, family life is concentrated on the weekend. "The three of us do a lot and try to make Elena happy," says the mother. She further describes that her daughter is inquisitive and sometimes very precocious, "she is like a big one." Elena always wants to know everything and also be there "when friends come to us."

\section{Interview with the patient and her mother}

A little later comes Elena, a slim, blond, pale and pretty girl with big blue eyes and a very serious expression on her face. She can barely break away from her mother and sits on her lap during the conversation, clutching her tightly. The mother tries again and again to free herself from the "clutches", which she hardly succeeds. The mother is clearly uncomfortable and seems annoyed. In the course of the conversation, 
Elena loosens her grip on her mother and corrects her quietly, but very decisively while speaking.

When the mother leaves the room, Elena curiously explores the room and begins to play in the dollhouse. "Look, this is what my room looks like, but I'm never in my room. I always sleep on a mattress in my parents' room. Mama always has to lie down with me, otherwise I won't fall asleep. Now I sleep in her bed too, it's more comfortable." Elena is very linguistically proficient, and one has the impression of talking to a much older girl, "a little lady", which is also reflected in her neat clothes (small chains, matching earrings, twinset). But when she starts to cry and to suck on her thumb and her cuddly toy, the tension between "adult" and "regressive" behavior becomes evident and her level of suffering becomes clearly noticeable.

\section{On psychodynamics, including the conflict of submission versus control}

In the conflict of Submission versus Control, there is a constant rebellion against obligations in the active mode, and submission and subordination in the passive mode. I perceive it as a control behavior to have everything under control and to secure the object for her. This active mode of Submission versus Control conflict shows up on many levels, not only in the demand for accompaniment while sleeping, also the natural way in which the daughter mixes with the parents' concerns (going out; friends; traveling) and these central co-determined, while parents are degraded to eager helpers. The fear of not being able to sleep alone and the screaming serve Elena as primary gain from illness (restoring closeness to the mother). With the help of regressive behavior, she constantly demands a lot of attention from the mother, but also from the father.

The mother now reacts increasingly angry and negative ("she has to be more independent at this age and I don't want to be taken in anymore") and on the other hand seems not to be able to adequately limit the excessive control needs of her daughter and to show the generation limit. "The little boss", as Elena calls herself, also affects the parents' relationship. Elena has not yet learned to allow her parents an independent relationship, and both parents have allowed it. It can therefore be assumed that the parents are involved in a couple conflict, as they tolerated Elena's behavior for years. Due to the ambivalent toddler-like and the flirtatious Elena slips into the role of a "powerful mastermind" who can and is allowed to control everything. In fact, after the start of treatment, it became apparent that a massive couple conflict was in the background; the parents separated immediately after starting therapy, and the father moved out to live with his girlfriend.

\section{Conclusion}

The case studies illustrate that patient, who have the same diagnosis, can have very different conflicts. These conflicts should be delt with in the subsequent psychodynamic psychotherapy, so that the symptoms can be given up.

It is no coincidence that in the presented cases of girls with F93 diagnosis, a conflict between the parents was more or less clearly in the background. In spite of very different conflicts that hinder development, there is also an oedipal theme. The couple conflict is only hinted at very cautiously with Maud, but it is already noticeable with Elena in the initial discussions and led to the separation of the parents at the beginning of the therapy. Elena's therapy subsequently showed that Elena was used as a surrogate partner by her parents, as both were dissatisfied in their partnership. As a result of this substitute partnership, Elena remained as a "powerful object" between the parents. Elena's turn to her peers and the associated strivings for autonomy and demarcation from her mother were very difficult for them to bear. A detachment from her mother was partially not feasible for Elena, because the mother repeatedly regressively tied her to herself through new diseases. This symbiotic amalgamation with her daughter still needed the mother, who Elena used as a self-object. She saw the detachment of her daughter as a loss, like the loss of her husband.

\section{Reference}

1. Hogrefe (2020) Task Force OPD-CA-2 (Ed.) OPD-CA-2 - Operationalized psychodynamic diagnosis: Manual of diagnosis and treatment planning.

Copyright: (C2020 Seiffge-Krenke I. This is an open-access article distributed under the terms of the Creative Commons Attribution License, which permits unrestricted use, distribution, and reproduction in any medium, provided the original author and source are credited. 